\title{
The contribution of mindfulness to outpatient substance use disorder treatment in Brazil: a preliminary study
}

\author{
Mayra P. Machado, ${ }^{1}$ iD Thiago M. Fidalgo, ${ }^{2}$ iD Silvia Brasiliano, ${ }^{3}$ Patrícia B. Hochgraf, ${ }^{3}$ Ana R. Noto ${ }^{1}$ \\ ${ }^{1}$ Departamento de Psicobiologia, Universidade Federal de São Paulo (UNIFESP), São Paulo, SP, Brazil. ${ }^{2}$ Departamento de Psiquiatria, \\ UNIFESP, São Paulo, SP, Brazil. ${ }^{3}$ Programa da Mulher Dependente Química (PROMUD), Departamento e Instituto de Psiquiatria (IPq), \\ Hospital das Clínicas, Faculdade de Medicina, Universidade de São Paulo (USP), São Paulo, SP, Brazil.
}

\begin{abstract}
Objective: To explore the contribution of a mindfulness-based intervention as an adjuvant to outpatient substance use disorder treatment. Outcomes included substance use behavior, depression and anxiety symptoms, and anger expression.

Methods: This preliminary study for a pragmatic randomized controlled trial with stratified random allocation included three months of follow-up. In two outpatient clinics linked to public universities, a mindfulness-based intervention plus treatment as usual (experimental group $n=22$ ) was compared to treatment as usual (control group $n=20$ ). The study included data from self-report measurements and the patients' records, which were evaluated according to intention-to-treat analysis through generalized estimating equations and generalized method of moments estimation.

Results: The experimental group had lower symptoms of depression $(b=-6.82 ; 95 \% \mathrm{Cl}-12.45$ to -1.18$)$ and anxiety $(b=-0.25 ; 95 \% \mathrm{Cl}-0.42$ to -0.09$)$, and anger expression $(b=-9.76 ; 95 \% \mathrm{Cl}-18.98$ to -0.54 ) three months after the intervention. We detected no effect on substance use behavior.

Conclusion: The mindfulness-based intervention yielded promising results as an adjuvant to outpatient substance use disorder treatment, since it reduced levels of highly prevalent symptoms in this population. However, further studies with longer follow-up periods and larger samples are required.
\end{abstract}

Keywords: Mindfulness; substance use disorder; outpatient care; comorbidity, meditation

\section{Introduction}

Substance use disorders (SUD) are defined as dysfunctional patterns of psychoactive substance use that encompass a set of cognitive, behavioral and physiological symptoms. ${ }^{1}$ There is a high prevalence of anxiety and depression in this population. According to a systematic review and meta-analysis, approximately $30 \%$ of people with alcohol or other drug use disorders also have an anxiety disorder and $20 \%$ had major depression in the past year. ${ }^{2}$ Anger, which is associated with violence, ${ }^{3}$ is of great importance, considering that over $70 \%$ of people in SUD treatment reported having suffered and/or perpetrated violence. ${ }^{4}$ Anxiety, depression and anger may lead to stronger craving responses and, together with other negative affect states, are the main predictors of substance use. ${ }^{5}$

Mindfulness-based interventions (MBI) seem promising for SUD treatment, and evidence has been found of their effectiveness. ${ }^{6}$ Mindfulness is the ability to focus attention on the present with a curious, kind, and non-judgmental attitude. ${ }^{7}$ It aims to increase awareness of triggers, habitual patterns and automatic reactions, and promotes

Correspondence: Mayra Pires Alves Machado, Rua Botucatu, 862, $1^{\circ}$ andar, Vila Clementino, CEP 04023-062, São Paulo, SP, Brazil. E-mail: mayra.pamachado@gmail.com

Submitted Jan 31 2020, accepted Feb 15 2020, Epub Jun 122020. the development of skillful action. ${ }^{8}$ Clinical trials with long follow-up indicate that $\mathrm{MBI}$ has several benefits for individuals with SUD, such as decreased consumption behavior, cravings and risk of relapse..$^{8-10}$

To our knowledge, no Brazilian studies have evaluated the effects of $\mathrm{MBI}$ on individuals being treated for different kinds of SUD. The objective of this preliminary study is to explore how an MBI called Mindfulness-based Relapse Prevention (MBRP) contributes to the outpatient SUD treatment. The outcomes were substance use behavior, anxiety and depression symptoms, and anger expression.

\section{Methods}

\section{Setting, participants and procedures}

This is a preliminary study for a pragmatic randomized controlled trial with stratified random allocation by gender and SUD type. During three months of follow-up, the MBI plus treatment as usual (experimental group $n=22$ ) was compared with treatment as usual (control group $n=20$ ). Treatment as usual includes occupational therapy, psychological, psychiatric, clinical and nutritional treatment, as well as guidance from a social worker.

How to cite this article: Machado MP, Fidalgo TM, Brasiliano S, Hochgraf PB, Noto AR. The contribution of mindfulness to outpatient substance use disorder treatment in Brazil: a preliminary study. Braz J Psychiatry. 2020;42:527-531. http://dx.doi.org/10.1590/1516-44462019-0725 
We recruited 42 patients in SUD treatment from two outpatient clinics linked to public universities in the city of São Paulo that offer multidisciplinary treatment for individuals with SUD. The inclusion criteria were a diagnosis of SUD, having been in SUD treatment for at least a month, literacy, and being over 18 years of age. The exclusion criteria were psychotic disorders, severe cognitive impairment, or suicidal ideation. Measurements were taken at baseline (T0), post-intervention (T1) and three months post-intervention (T2). The percentage of patients remaining in treatment at $\mathrm{T} 1$ was $70 \%$ in the control group and $68.2 \%$ in the experimental group. The percentage of patients remaining in treatment at T2 was $25 \%$ in the control group and $54.5 \%$ in the experimental group. The main reasons that for dropout were that the participants changed residence, could not be found, relapsed, or didn't fill out the questionnaires.

\section{Intervention}

The intervention, MBRP, consisted of eight two-hour weekly sessions of mindfulness meditation and psychoeducational activities. ${ }^{8}$ It was conducted by a trained instructor with expertise in both MBRP and SUD.

\section{Outcomes and measurements}

We used questionnaires and the patients' records to obtain data on sociodemographics and SUD. All other instruments assessing outcomes were self-report. Substance use behavior was assessed with the Timeline Followback Method. ${ }^{11}$ Anger expression was assessed with the Anger Expression Index, which includes 32 items from the State-Trait Anger Expression Inventory (STAXI2) $(\alpha=0.91){ }^{12}$ Depression was assessed with the Center for Epidemiologic Studies - Depression scale, which includes 20 items $(\alpha=0.93) .{ }^{13}$ Trait anxiety was assessed with the Trait subscale of the State-Trait Anxiety Inventory, which includes 20 items $(\alpha=0.93) .{ }^{14}$

\section{Statistical analysis}

We carried out descriptive analyses and normality testing through histogram graphs, percentiles, kurtosis and skewness, followed by the $t$-test for continuous variables and the chi-square test for categorical variables. We estimated the effects of the intervention using intentionto-treat analysis with generalized method of moments estimation and generalized estimating equations. Hence, all randomized individuals were considered in the final analyses. Due to the small sample size, we could not perform adjusted or stratified analyses by gender or SUD type.

\section{Ethics statement}

The Universidade Federal de São Paulo (UNIFESP) research ethics committee approved the project (number 1.346.744).

\section{Results}

The sample included the same number of men and women, and the mean age was $44 \pm 11.2$ years. More than half the sample had SUD for multiple substances, reaching $63.6 \%$ in the experimental group. The second most prevalent SUD type was alcohol in the experimental group $(22.7 \%)$, and cocaine/crack in the control group $(20 \%)$. There were no statistically significant differences in the outcomes between the experimental and the control groups at baseline.

Table 1 and Figure 1 show the effects of the intervention over time. While the intervention had no effect on substance use behaviors, the experimental group's depression and anxiety symptoms and anger expression scores reduced significantly between T0 and T2, with a detectable decrease in anger expression between TO and $\mathrm{T} 1$.

\section{Discussion}

This study has provided evidence that MBI contributes to outpatient SUD treatment. Although the dropout rates at T2 were higher than those of previous studies, ${ }^{8,9}$ these studies provided monetary incentives to participants. This approach can enhance adherence but is not allowed under Brazilian law.

Although MBRP had no effect on substance use behavior, it significantly reduced the other outcomes. Bowen et al. ${ }^{8}$ also found that MBRP had no effects on consumption behavior in three months of follow-up. However, they found that alcohol consumption was reduced after six months, and consumption of other substances was lower after one year of follow-up. Witkiewitz \& Bowen ${ }^{9}$ found that MBRP was associated with fewer days of substance consumption after 15 weeks of follow-up. Moreover, it should be pointed out that the participants in our sample reported greater substance consumption at baseline than those in the two abovementioned studies. Moreover, in both of these studies, MBRP was applied post-treatment during the maintenance period, rather than as an adjunct to outpatient treatment.

In the present study, MBRP significantly reduced scores for both depression and anxiety symptoms three months after the intervention. These results add to the evidence of a recent systematic review and metaanalysis ${ }^{15}$ that evaluated the effects of MBI on different treatment outcomes in individuals with SUD. No studies were found that evaluated the effects of MBRP on anxiety or anger. Additionally, a study ${ }^{10}$ that estimated the mediating effect of craving on the relationship between depression and substance use found that this mediation ceased to exist in participants who received MBRP, as did the association between depression and craving, although it detected no decrease in depression scores.

These findings are consistent with the objective of MBRP, which is to change the way individuals relate to challenging emotions and, thus, increase skillful responses. $^{8}$ Furthermore, in the present study, anxiety and depression symptoms decreased in the MBRP group, 
Table 1 Analysis of the effects of mindfulness-based relapse prevention on the outcomes over time

\begin{tabular}{|c|c|c|c|c|c|}
\hline & & & T0* & $\mathrm{T} 1^{\dagger}$ & $\mathrm{T}^{\ddagger}$ \\
\hline & $\mathrm{b}(95 \% \mathrm{Cl})$ & Group & $M \pm S D(n)$ & $M \pm S D(n)$ & $M \pm S D(n)$ \\
\hline Depression & & & & & \\
\hline Group (ref = control) & $-4.95(-13.63$ to 3.72$)$ & & & & \\
\hline T1 $($ ref = baseline) & $0.79(-6.63$ to 8.21$)$ & Control & $28.0 \pm 14.4(20)$ & $30.2 \pm 14.9(15)$ & $27.1 \pm 14.6(6)$ \\
\hline T2 (ref = baseline) & $-3.98(-8.37$ to 0.40$)$ & & & & \\
\hline Group vs. T1 (ref = control vs. baseline) & $-3.51(-12.50$ to 5.47$)$ & Experimental & $23.0 \pm 13.7(21)$ & $20.8 \pm 8.3(13)$ & $12.4 \pm 6.6(10)$ \\
\hline Group vs. T2 (ref = control vs. baseline) & $-6.82(-12.45$ to -1.18$)$ & & & & \\
\hline Anxiety & & & & & \\
\hline Group (ref = control) & $-0.06(-0.26$ to 0.14$)$ & & & & \\
\hline $\mathrm{T} 1$ (ref = baseline) & $0.01(-0.07$ to 0.11$)$ & Control & $53.2 \pm 16.1(14)$ & $54.2 \pm 16.8(11)$ & $55.3 \pm 17.2(6)$ \\
\hline T2 (ref = baseline) & $0.02(-0.09$ to 0.13$)$ & & & & \\
\hline Group vs. T1 (ref = control vs. baseline) & $-0.05(-0.20$ to 0.08$)$ & Experimental & $50.0 \pm 10.9(14)$ & $48.1 \pm 14.2(8)$ & $36.5 \pm 9.2(7)$ \\
\hline Group vs. T2 (ref = control vs. baseline) & $-0.25(-0.42$ to -0.09$)$ & & & & \\
\hline Anger expression & & & & & \\
\hline Group (ref = control) & $2.92(-8.13$ to 13.98$))$ & & & & \\
\hline $\mathrm{T} 1$ (ref = baseline) & $0.56(-4.33$ to 5.46$)$ & Control & $45.0 \pm 16.4(14)$ & $45.6 \pm 14.2(12)$ & $40.3 \pm 8.7(6)$ \\
\hline T2 (ref = baseline) & $-1.94(-8.60$ to 4.70$)$ & & & & \\
\hline Group vs. T1 (ref = control vs. baseline) & $-10.65(-17.67$ to -3.63$)$ & Experimental & $47.0 \pm 17.3(17)$ & $37.6 \pm 12.8(9)$ & $32.5 \pm 14.9(6)$ \\
\hline Group vs. T2 (ref = control vs. baseline) & $-9.76(-18.98$ to -0.54$)$ & & & & \\
\hline Alcohol consumption, standard doses & & & & & \\
\hline Group $($ ref = control) & $1.37(-0.07$ to 2.82$)$ & & & & \\
\hline T1 (ref = baseline) & $-0.09(-1.34$ to 1.20$)$ & Control & $0.3 \pm 0.6(20)$ & $0.2 \pm 0.5(16)$ & $0.4 \pm 0.7(15)$ \\
\hline T2 (ref = baseline) & $0.39(-0.77$ to 1.54$)$ & & & & \\
\hline Group vs. T1 (ref = control vs. baseline) & $0.08(-1.30$ to 1.47$)$ & Experimental & $1.5 \pm 3.0(22)$ & $1.6 \pm 2.6(20)$ & $1.3 \pm 2.7(15)$ \\
\hline Group vs. T2 (ref = control vs. baseline) & $-0.73(-2.01$ to 0.56$)$ & & & & \\
\hline$\%$ of days with heavy alcohol use & & & & & \\
\hline Group (ref = control) & $1.27(-0.55$ to 3.08$)$ & & & & \\
\hline $\mathrm{T} 1$ (ref = baseline) & $-0.36(-0.94$ to 0.21$)$ & Control & $3.1 \pm 7.1(20)$ & $1.2 \pm 4.0(16)$ & $2.8 \pm 5.0(15)$ \\
\hline T2 (ref = baseline) & $0.41(-0.06$ to 0.88$)$ & & & & \\
\hline Group vs. T1 (ref = control vs. baseline) & $0.57(-0.03$ to 1.18$)$ & Experimental & $10.4 \pm 23.2(22)$ & $12.4 \pm 23.8(20)$ & $11.7 \pm 25.7(15)$ \\
\hline Group vs. T2 (ref = control vs. baseline) & $-0.35(-0.87$ to 0.16$)$ & & & & \\
\hline Marijuana consumption, cigarettes & & & & & \\
\hline Group (ref = control) & $1.32(-0.81$ to 3.47$)$ & & & & \\
\hline $\mathrm{T} 1$ (ref = baseline) & $-0.60(-2.60$ to 1.39$)$ & Control & $0.2 \pm 0.6(20)$ & $0.9 \pm 0.2(16)$ & $0.1 \pm 0.3(15)$ \\
\hline T2 (ref = baseline) & $-0.55(-2.54$ to 1.42$)$ & & & & \\
\hline Group vs. T1 (ref = control vs. baseline) & $0.28(-1.84$ to 2.41$)$ & Experimental & $0.7 \pm 2.3(22)$ & $0.6 \pm 1.6(20)$ & $0.4 \pm 0.1(15)$ \\
\hline Group vs. T2 (ref = control vs. baseline) & $-0.20(-2.40$ to 1.98$)$ & & & & \\
\hline$\%$ of days with cocaine/crack use & & & & & \\
\hline Group (ref = control) & $-0.54(-13.7$ to 12.6$)$ & & & & \\
\hline $\mathrm{T} 1$ (ref = baseline) & $-1.36(-8.3$ to 5.5$)$ & Control & $9.8 \pm 24.0(20)$ & $7.9 \pm 21.4(16)$ & $5.0 \pm 9.2(15)$ \\
\hline T2 (ref = baseline) & $-4.73(-11.8$ to 2.35$)$ & & & & \\
\hline Group vs. T1 (ref = control vs. baseline) & $6.59(-2.72$ to 15.9$)$ & Experimental & $9.3 \pm 23.7(22)$ & $15.2 \pm 27.5(20)$ & $4.5 \pm 10.0(15)$ \\
\hline Group vs. T2 (ref = control vs. baseline) & $5.61(-4.32$ to 15.5$)$ & & & & \\
\hline
\end{tabular}

$95 \% \mathrm{Cl}=95 \%$ confidence interval; $\mathrm{M}=$ mean; $\mathrm{n}=$ sample size; $\mathrm{SD}=$ standard deviation.

* Information on substance consumption based on the 15 prior to T0.

Information on substance consumption based on the period between T0 and T1.

$\$$ Information on substance consumption based on the period between T1 and T2.

Bold type denotes statistical significance $(p<0.05)$.

showing their altered emotional state. However, studies with longer follow-ups are required to determine whether this improvement is maintained and its possible impact on consumption behavior.

Finally, MBRP significantly reduced anger expression indices. Given their prevalence, addressing violence and anger issues in SUD treatment centers is fundamental, ${ }^{4}$ and health teams require better training to do so. In this respect, MBRP seems quite promising, since it offers an effective alternative for indirectly dealing with anger and could also reduce violence. However, new studies are required to corroborate this hypothesis.
One major strength of this study is that it is the first in Brazil to investigate the contribution of $\mathrm{MBI}$ to SUD treatment, finding positive results for the patients' mental health. This is also the first study to evaluate the effect of MBRP on anger expression in a population with SUD. Another important issue is that the intervention was used as an adjuvant to outpatient treatment, evaluating MBRP in the context of a treatment already under way, which favors external validity. On the other hand, the follow-up was relatively short and could not detect changes in outcomes that, according to previous studies, require longer follow-up, such as substance use behavior. 

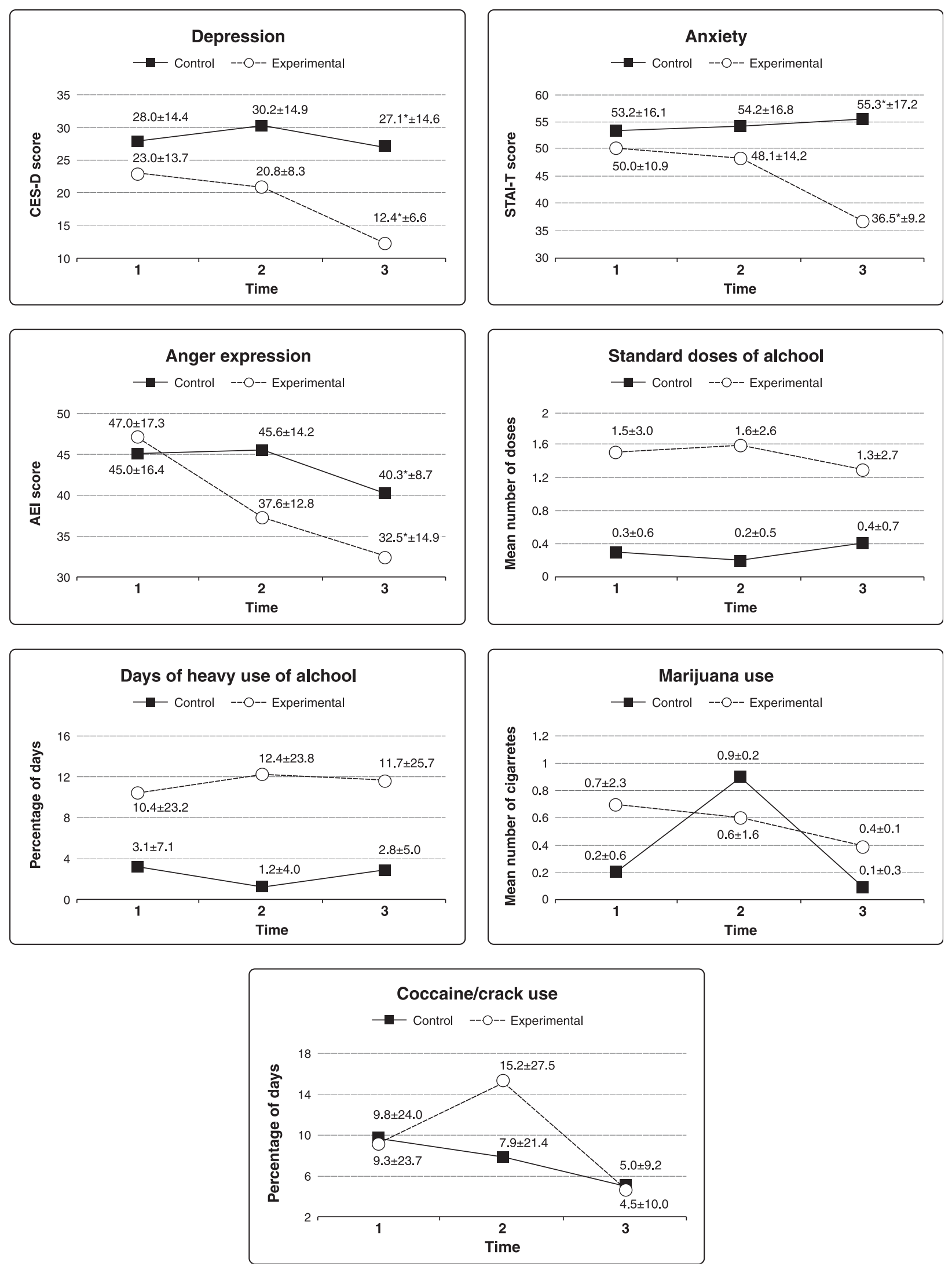

Figure 1 Depression, anxiety and anger expression scores (mean \pm standard deviation) of both groups over time. CES-D = Center for Epidemiologic Studies - Depression scale; STAI-T = State-Trait Anxiety Inventory - Trait subscale; AEI = Anger Expression Index. $* p<0.05$ using baseline parameters as reference values. 
Although the sample size was large enough for a preliminary study, it did not allow analysis of consumption behavior stratified by substance type or gender or adjustment for other variables. The lack of data on home behavior and the high dropout rates are another limitation.

This study has provided evidence of the potential benefits of $\mathrm{MBI}$ when applied alongside outpatient SUD treatment, considering important mental health outcomes. However, the high dropout rate suggests that the protocol should be tested in other formats. Given that in 2017 the Brazilian National Policy on Integrative and Complementary Practices included meditation, further research is necessary to promote evidence-based practices.

\section{Acknowledgements}

The authors would like to thank all of the participants, as well as the clinical teams of the outpatient facilities. We are also thankful to Fundação de Amparo à Pesquisa do Estado de São Paulo (FAPESP; grant 2015/19472-5), the Conselho Nacional de Desenvolvimento Científico e Tecnológico (CNPq; process 142267/2015-5), and the Associação de Fundo e Incentivo à Pesquisa (AFIP) for the financial resources, materials and infrastructure that made this research possible.

\section{Disclosure}

The authors report no conflicts of interest.

\section{References}

1 American Psychiatric Association. Diagnostic and Statistical Manual of Mental Disorders, Fifth Edition (DSM-5). Arlington: American Psychiatric Publishing; 2013.

2 Lai HM, Cleary M, Sitharthan T, Hunt GE. Prevalence of comorbid substance use, anxiety and mood disorders in epidemiological surveys, 1990-2014: a systematic review and meta-analysis. Drug Alcohol Depend. 2015;154:1-13.
3 Sprunger JG, Eckhardt Cl, Parrot DJ. Anger, problematic alcohol use, and intimate partner violence victimisation and perpetration. Crim Behav Ment Health. 2015;25:237-86.

4 Gilchrist G, Radcliffe P, Noto AR, d'Oliveira AF. The prevalence and factors associated with ever perpetrating intimate partner violence by men receiving substance use treatment in Brazil and England: a cross-cultural comparison. Drug Alcohol Rev. 2017;36:34-51.

5 Olsson KL, Cooper RL, Nugent WR, Reid RC. Addressing negative affect in substance use relapse prevention. J Hum Behav Soc Environ. 2016;26:2-14.

6 Li W, Howard MO, Garland EL, McGovern P, Lazar M. Mindfulness treatment for substance misuse: a systematic review and metaanalysis. J Subst Abuse Treat. 2017;75:62-96.

7 Kabat-Zinn J. Full catastrophe living: using the wisdom of your body and mind to face stress, pain, and illness. New York: Delta Trade Paperbacks; 1990.

8 Bowen S, Witkiewitz K, Clifasefi SL, Grow J, Chawla N, Hsu SH, et al. Relative efficacy of mindfulness-based relapse prevention, standard relapse prevention, and treatment as usual for substance use disorders. JAMA Psychiatry. 2014;71:547-56.

9 Witkiewitz K, Warner K, Sully B, Barricks A, Stauffer C, Thompson $\mathrm{BL}$, et al. Randomized trial comparing mindfulness-based relapse prevention with relapse prevention for women offenders at a residential addiction treatment center. Subst. Use Misuse. 2014;49: 536-46.

10 Witkiewitz K, Bowen S. Depression, craving and substance use following a randomized trial of mindfulness-based relapse prevention. J Consult Clin Psychol. 2010;78:362-74.

11 Fals-Stewart W, Farrel TJ, Freitas TT, McFarlin SK, Rutigliano P. The timeline followback reports of psychoatctive substance use by drugabusing patients: psychometric properties. J Consult Clin Psychol. 2000;68:134-44.

12 Spielberger CD. STAXI-2: Inventário de expressão da raiva como estado e traço. São Paulo: Vetor; 2010.

13 Batistoni SST, Neri AL, Cupertino APFB. Validade da escala de depressão do Center for Epidemiological Studies entre idosos brasileiros. Rev Saude Publica. 2007;41:598-605.

14 Biaggio A, Natalício L, Spielberger CD. Desenvolvimento da forma experimental em português do Inventário de Ansiedade TraçoEstadp (IDATE), de Spielberger. Arq Bras Psicol Apl. 1977;29: 31-44.

15 Cavicchioli M, Movalli M, Maffei C. The clinical efficacy of mindfulness-based treatments for alcohol and drugs use disorders: a meta-analytic review of randomized and nonrandomized controlled trials. Eur Addict Res. 2018;24:137-62. 